\title{
Inhibition of Cyclooxygenase-2 Suppresses the Recruitment of Endothelial Progenitor Cells in the Microvasculature of Endometriotic Lesions
}

\author{
Jeannette Rudzitis-Auth, Ruth M. Nickels, Michael D. Menger, and Matthias W. Laschke
}

From the Institute for Clinical \& Experimental Surgery, Saarland University, Homburg/Saar, Germany

\author{
Accepted for publication \\ October 3, 2017. \\ Address correspondence to \\ Jeannette Rudzitis-Auth, Ph.D., \\ Institute for Clinical \& \\ Experimental Surgery, Saarland \\ University, D-66421 Homburg/ \\ Saar, Germany. E-mail: \\ jeannette.rudzitis-auth@uks.eu.
}

\begin{abstract}
The incorporation of endothelial progenitor cells (EPCs) into newly developing blood vessels contributes to the vascularization of endometriotic lesions. We analyzed whether cyclooxygenase (COX)-2 signaling regulates this vasculogenic process. Endometriotic lesions were surgically induced in irradiated FVB/N mice, which were reconstituted with bone marrow from FVB/N-TgN [Tie2/green fluorescent protein (GFP)] 287 Sato mice. The animals received $\beta$-estradiol 17 -valerate once a week and were treated daily with the selective COX-2 inhibitor parecoxib $(25 \mathrm{mg} / \mathrm{kg}$ ) or vehicle (control) for 7 and 28 days. Analyses involved the determination of lesion growth, cyst formation, homing of $\mathrm{GFP}^{+} / \mathrm{Tie}^{+} \mathrm{EPC}$, numbers of circulating EPCs, vascularization, cell proliferation, apoptosis, and immune cell infiltration by means of highresolution ultrasonography, caliper measurements, flow cytometry, histologic analysis, and immunohistochemical analysis. In parecoxib-treated mice, blood circulating EPCs were higher, but numbers of recruited EPCs in endometriotic lesions were significantly lower when compared with controls. This finding was associated with an impaired early vascularization and stromal tissue growth as well as reduced glandular secretory activity of the lesions. Parecoxib-treated lesions further contained less proliferating and more apoptotic cells and exhibited lower numbers of infiltrating macrophages and neutrophilic granulocytes. These findings demonstrate that the inhibition of COX-2 suppresses vasculogenesis in endometriotic lesions, which may contribute to an impaired lesion vascularization and growth. (Am J Pathol 2018, 188: 450-460; https://doi.org/10.1016/j.ajpath.2017.10.013)
\end{abstract}

Endometriosis is a painful gynecologic disease, which is characterized by the presence of endometriotic lesions in the peritoneal cavity. ${ }^{1}$ The survival, proliferation, and spread of the endometrial-like tissue within these lesions are dependent on sufficient vascularization. ${ }^{2,3}$ Accordingly, dense microvascular networks are a typical feature of developing endometriotic lesions. ${ }^{4}$ Moreover, endometriosis is associated with the up-regulation of multiple proangiogenic factors and cytokines that can be detected in high concentrations in the peritoneal fluid of patients with endometriosis. ${ }^{5}$ Hence, targeting the microvasculature of endometriotic lesions is increasingly suggested as a novel therapeutic strategy in the treatment of the disease. ${ }^{6,7}$

Blood vessel formation in endometriotic lesions involves different cellular mechanisms. Driven by the hypoxiainduced release of angiogenic growth factors, vascular sprouts from vessels of the surrounding tissue progressively grow into the engrafting lesions, where they interconnect with each other and finally form blood-perfused microvascular networks. ${ }^{8,9}$ Besides this angiogenic process, circulating endothelial progenitor cells (EPCs) from the bone marrow contribute to the lesions' vascularization by incorporation into the endothelial lining of newly developing microvessels, also referred to as vasculogenesis. ${ }^{10-13}$ Angiogenesis and vasculogenesis in endometriotic lesions are dependent on estrogen stimulation. ${ }^{8,14}$ However, although various mechanisms that mediate angiogenesis in endometriosis have already been identified, ${ }^{15-17}$ the regulation of vasculogenesis in endometriotic lesions remains largely unknown.

Cyclooxygenase (COX)-2, the rate-limiting enzyme of the prostaglandin (PG) synthesis pathway, determines the

Supported by Deutsche Forschungsgemeinschaft (German Research Foundation) grant LA 2682/3-1 (M.W.L.).

Disclosures: None declared. 
A

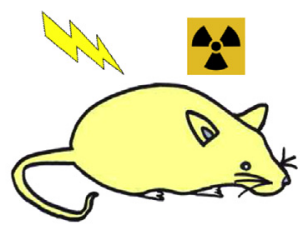

C

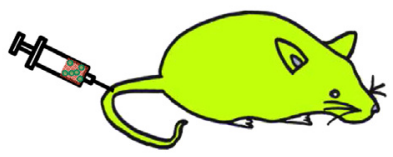

$\mathbf{E}$

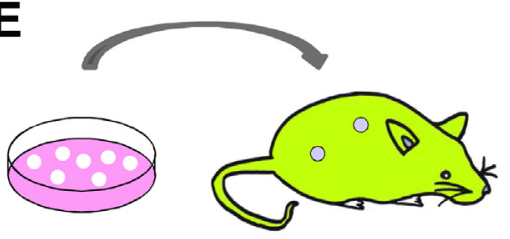

B

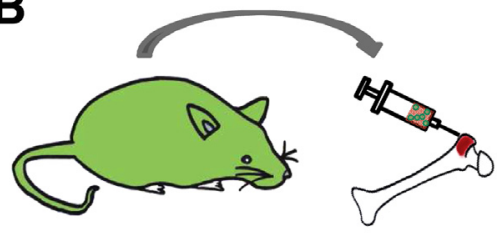

D

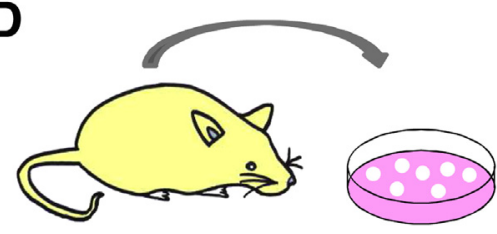

$\mathbf{F}$

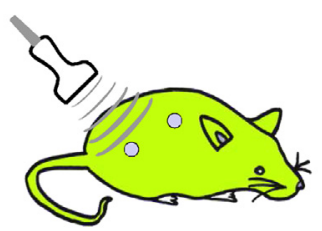

Figure 1 Schematic illustration of the murine green fluorescent protein $(\mathrm{GFP})^{+} / \mathrm{GFP}^{-}$crossover design model, which enables the quantitative assessment of $\mathrm{GFP}^{+} / \mathrm{Tie}^{+}$ endothelial progenitor cells in the microvasculature of $\mathrm{GFP}^{-}$endometriotic lesions. A: Irradiation of $\mathrm{FB} / \mathrm{N}$ mice. B: Isolation of bone marrow cells from $\mathrm{FVB} / \mathrm{N}-\mathrm{TgN}$ (Tie/GFP) 287 Sato mice. C: Injection of $2 \times 10^{7}$ bone marrow cells into irradiated $\mathrm{FVB} / \mathrm{N}$ mice. D: Isolation of uterine tissue samples from FVB/N donor mice. E: Induction of endometriotic lesions in bone marrow-reconstituted mice. F: Repetitive in vivo analysis of endometriotic lesions during 28 days. proliferative activity and viability of EPCs and, thus, represents a potential target for the inhibition of tumor vasculogenesis. ${ }^{18}$ Of interest, COX-2 is also highly expressed in endometriosis, ${ }^{19,20}$ and the application of selective COX-2 inhibitors has been suggested as an option for the management of endometriosis-associated pain. ${ }^{21,22}$ Experimental studies further indicate that the inhibition of COX-2 reduces the vascularization of endometriotic lesions. ${ }^{23,24}$ This reduction is partly caused by the suppression of vascular endothelial growth factor (VEGF)-mediated angiogenesis. ${ }^{24}$ On the other hand, it may be speculated that targeting COX-2 signaling also affects vasculogenesis in endometriosis.

To test this hypothesis, we surgically induced endometriotic lesions in a previously established murine green fluorescent protein $(\mathrm{GFP})^{+} / \mathrm{GFP}^{-}$crossover design model. ${ }^{14}$ This model allowed the quantitative assessment of $\mathrm{GFP}^{+}$/ $\mathrm{Tie}^{+}$EPCs in the microvasculature of $\mathrm{GFP}^{-}$endometriotic lesions within mice, which were treated with the selective COX-2 inhibitor parecoxib or vehicle.

\section{Materials and Methods}

\section{Animals}

Twelve- to 16-week-old female FVB/N mice (Institute for Clinical \& Experimental Surgery, Homburg/Saar, Germany) and transgenic FVB/N-TgN (Tie2/GFP) 287 Sato mice (Jackson Laboratories, Bar Harbor, ME) with a body weight of 20 to $25 \mathrm{~g}$ were used. The animals were housed in open cages ( $n=4$ to 5 per cage) under a 12-hour day/night cycle and had access to water and standard pellet food ad libitum (Altromin, Lage, Germany).
All experiments were performed according to the German legislation on protection of animals and the NIH's Guide for the Care and Use of Laboratory Animals ${ }^{25}$ and were approved by the local governmental animal care committee (Landesamt für Verbraucherschutz, Saarland, Germany; permission number: 25/2012).

\section{Bone Marrow Transplantation}

To generate $\mathrm{GFP}^{+} / \mathrm{Tie}^{+}$chimeras, $37 \mathrm{FVB} / \mathrm{N}$ mice were lethally irradiated by exposure to a single dose of $8.5 \mathrm{~Gy}$ (Figure 1A). After 4 hours, they received the bone marrow of $19 \mathrm{FVB} / \mathrm{N}-\mathrm{TgN}$ (Tie2/GFP) 287 Sato mice. For this purpose, the donor mice were sacrificed, and the femurs and tibias were flushed with cold phosphate-buffered saline. Subsequently, $2 \times 10^{7}$ bone marrow cells resuspended in $300 \mu \mathrm{L}$ of cold phosphate-buffered saline were injected via the tail vein into the recipient animals, which were allowed to recover for 4 to 6 weeks for the reconstitution of their bone marrow (Figure 1, B and $\mathrm{C}$ ).

\section{Induction of Endometriotic Lesions}

Peritoneal endometriotic lesions were surgically induced by suturing uterine tissue samples from $19 \mathrm{FVB} / \mathrm{N}$ donor mice to the abdominal wall of $\mathrm{GFP}^{+} / \mathrm{Tie}^{+}$chimeras, as described previously. ${ }^{14}$ Before the induction of endometriotic lesions, estrus cycling of the donor mice was determined to exclude morphologic and functional differences among individual uterine tissue samples attributable to different sex hormone levels. Cytologic analysis of vaginal lavage samples was performed by pipetting $15 \mu \mathrm{L}$ of $0.9 \%$ saline into the vagina. The cell suspension was then transferred onto a glass slide and 

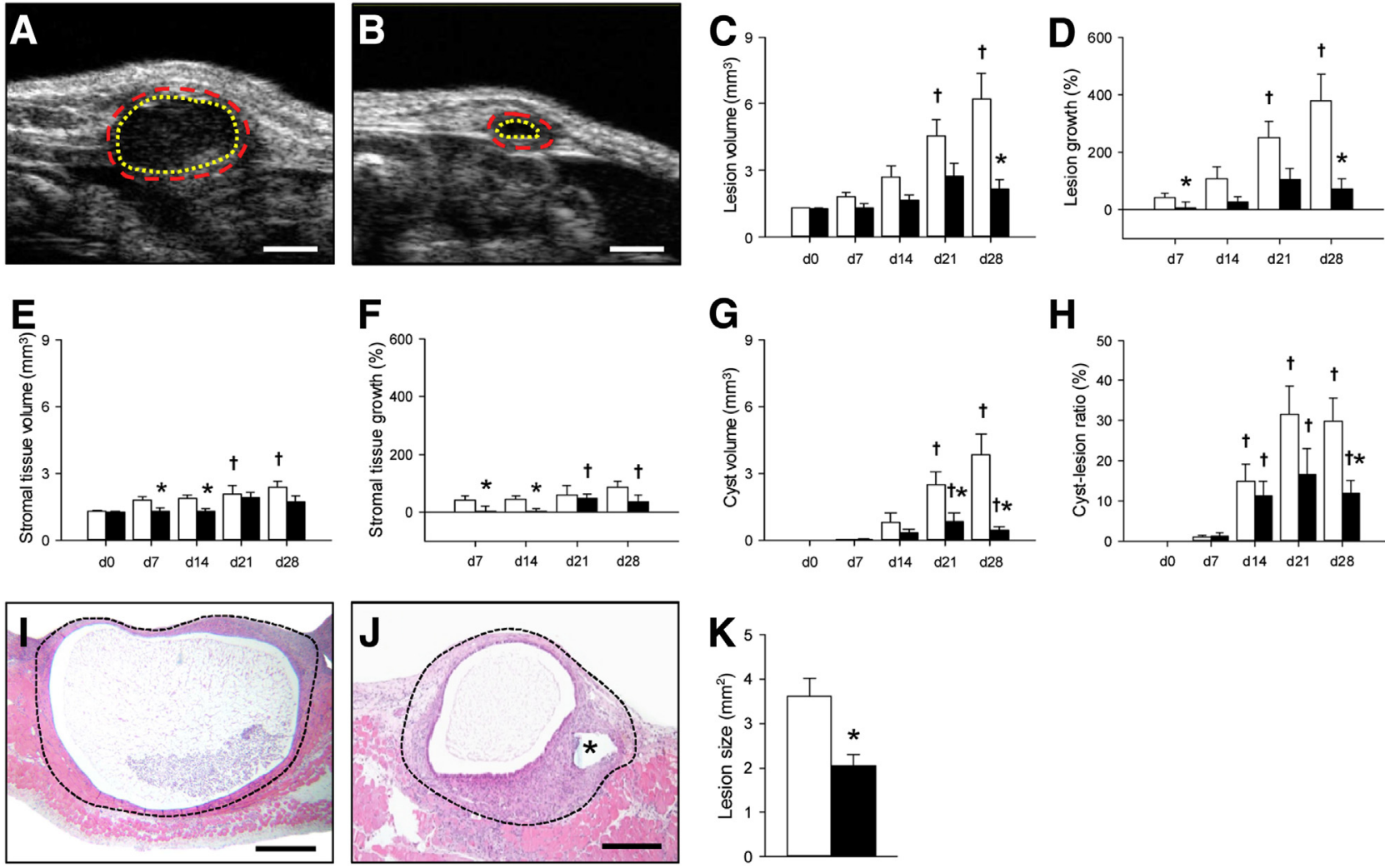

Figure 2 A and B: High-resolution ultrasonography of endometriotic lesions (borders marked by red dashed lines, cyst-like dilated glands marked by yellow dotted lines) 28 days after transplantation of uterine tissue samples to the abdominal wall of bone marrow-reconstituted FVB/N mice. The animals received $\beta$-estradiol 17-valerate once a week and were additionally treated with parecoxib $(\mathbf{B})$ or vehicle $(\mathbf{A})$ by daily i.p. injections. $\mathbf{C}-\mathbf{H}$ : 0 verall lesion volume $(\mathbf{C})$, lesion growth $(\mathbf{D})$, stromal tissue volume $(\mathbf{E})$, stromal tissue growth $(\mathbf{F})$, cyst volume $(\mathbf{G})$, and cyst/lesion ratio $(\mathbf{H})$ of endometriotic lesions of bone marrow-reconstituted FVB/N mice, which received $\beta$-estradiol 17 -valerate once a week and were additionally treated with parecoxib (black bars) or vehicle (white bars) by daily i.p. injections throughout an observation period of 28 days. I and J: Hematoxylin and eosin-stained endometriotic lesions (borders marked by dashed line; asterisk indicates suture) at day 28 after transplantation of uterine tissue samples into the peritoneal cavity of bone marrow-reconstituted FVB/N mice, which received $\beta$-estradiol 17-valerate once a week and were additionally treated with parecoxib (J) or vehicle (I) by daily i.p. injections. K: Caliper-assessed lesion size of endometriotic lesions of bone marrow-reconstituted FVB/N mice at day 28 . The animals received $\beta$-estradiol 17 valerate once a week and were additionally treated with parecoxib (black bar) or vehicle (white bar) by daily i.p. injections throughout an observation period of 28 days. Data are expressed as means \pm SEM. $n=9$ parecoxib-treated mice $(\mathbf{C}-\mathbf{H}, \mathbf{K}) ; n=10$ vehicle-treated mice $(\mathbf{C}-\mathbf{H}, \mathbf{K})$. ${ }^{*} P<0.05$ versus vehicle; ${ }^{\dagger} P<0.05$ versus day 0 . Scale bars: $1 \mathrm{~mm}$ (A and $\left.\mathbf{B}\right) ; 500 \mu \mathrm{m}$ (I and $\left.\mathbf{J}\right)$. d, days.

examined under a phase contrast microscope $(\mathrm{CH}-2$; Olympus, Hamburg, Germany) to identify animals in the stage of estrus as tissue donors for the experiments.

For harvesting of uterine tissue samples, the donor mice were anesthetized by an i.p. injection of $75 \mathrm{mg} / \mathrm{kg}$ of ketamine (Pharmacia GmbH, Erlangen, Germany) and $15 \mathrm{mg} / \mathrm{kg}$ of xylazin (Bayer, Leverkusen, Germany). The two uterine horns were excised after midline laparotomy and placed into a Petri dish that contained Dulbecco's modified Eagle medium ( $10 \%$ fetal calf serum, $100 \mathrm{U} / \mathrm{mL}$ of penicillin, $0.1 \mathrm{mg} / \mathrm{mL}$ of streptomycin; PAA, Cölbe, Germany). Then, 2-mm tissue samples were removed from the longitudinally opened uterine horns by means of a dermal biopsy punch (Stiefel Laboratorium $\mathrm{GmbH}$, Offenbach am Main, Germany) and microsurgical instruments (Figure 1D).

After laparotomizing the anesthetized $\mathrm{GFP}^{+} / \mathrm{Tie}^{+}$ recipient animals, one tissue sample was fixed with a 60 Prolene suture (Ethicon Products, Norderstedt, Germany) to the right and the left site of the abdominal wall
(Figure 1E). Subsequently, the laparotomy was closed with running 6-0 Prolene muscle and skin sutures. During the rest of the experiments, physiologic estrogen levels in the irradiated mice were achieved by s.c. injection of $100 \mu \mathrm{g} / \mathrm{kg}$ of $\beta$-estradiol 17 -valerate (dissolved in $100 \mu \mathrm{L}$ of corn oil; Sigma-Aldrich, Taufkirchen, Germany) once a week. ${ }^{14}$ To analyze the effect of COX-2 inhibition on the homing of EPCs in endometriotic lesions, the mice were additionally treated with the selective COX-2 inhibitor parecoxib (25 $\mathrm{mg} / \mathrm{kg} ; n=18$ ) or vehicle (sodium chloride; control; $n=19$ ) by daily i.p. injections.

\section{High-Resolution Ultrasonography and Analysis}

The fixed uterine tissue samples were repetitively analyzed with a Vevo 770 high-resolution ultrasonography system (VisualSonics, Toronto, ON, Canada) by means of a real-time microvisualization 704 Scanhead (VisualSonics) with a center frequency of $40 \mathrm{MHz}$ and a focal depth of $6 \mathrm{~mm}$ 
d7
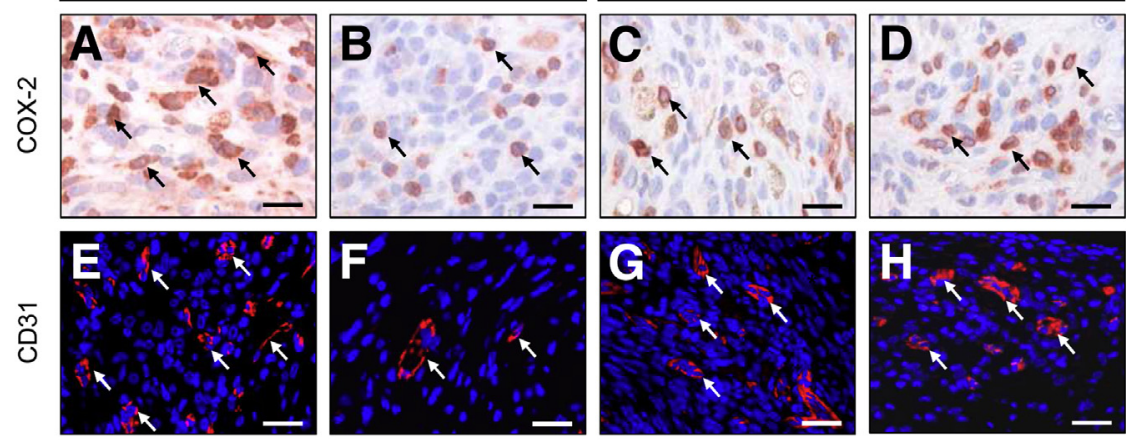

$\mathbf{I}_{50}$
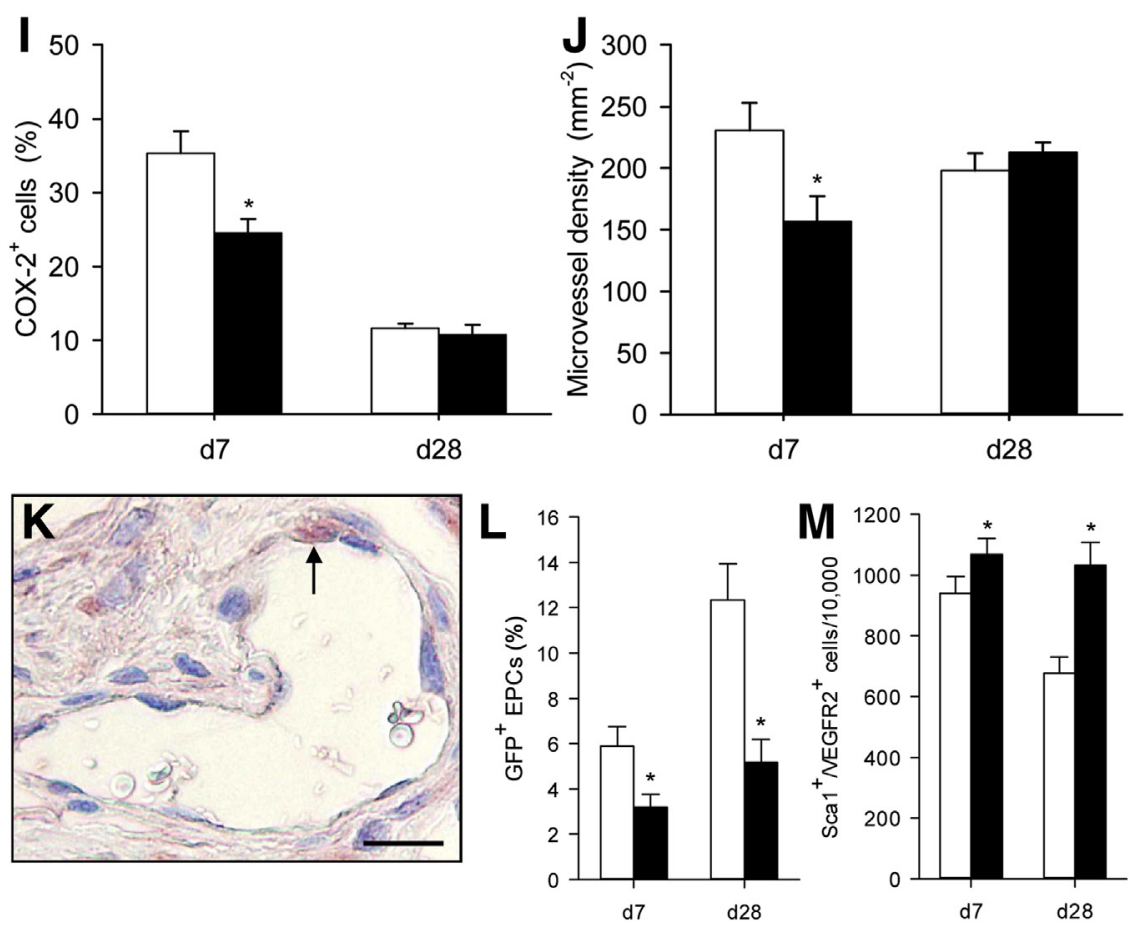

Figure 3 A-H: Immunohistochemical and immunofluorescent detection of cyclooxygenase (COX) $-2^{+}$cells (A-D, arrows) and microvessels $(\mathbf{E}-\mathbf{H}$, arrows) in the stroma of endometriotic lesions at day 7 (A, B, E, and F) and 28 (C, D, G, and H) after transplantation of uterine tissue samples into the peritoneal cavity of bone marrow -reconstituted FVB/N mice, which received $\beta$ estradiol 17-valerate once a week and were additionally treated with parecoxib (B, D, F, and $\mathbf{H})$ or vehicle (A, C, E, and $\mathbf{G})$ by daily i.p. injections. The immunofluorescent sections $(\mathbf{E}-\mathbf{H})$ were stained with Hoechst 33342 to identify cell nuclei (blue) and an antibody against CD31 for the detection of microvessels (red). I and J: COX-2 ${ }^{+}$cells (I) and microvessel density $(\mathbf{J})$ in the stroma of endometriotic lesions of bone marrow-reconstituted FVB/N mice, which received $\beta$-estradiol 17-valerate once a week and were additionally treated with parecoxib (black bars) or vehicle (white bars) by daily i.p. injections throughout an observation period of 7 and 28 days. K: Immunohistochemical detection of a green fluorescent protein (GFP) ${ }^{+}$ endothelial progenitor cell (EPC) (arrow) incorporated in the microvascular endothelium of a vehicletreated endometriotic lesion at day 28. $\mathbf{L}$ and $\mathbf{M}$ : $\mathrm{GFP}^{+}$EPCs (L) of endometriotic lesions and number of stem cell antigen- $1^{+} /$vascular endothelial growth factor receptor (VEGFR)-2 ${ }^{+}$EPCs per 10,000 events in the lymphocyte population of blood (M) of bone marrow-reconstituted FVB/N mice, which received $\beta$-estradiol 17-valerate once a week and were additionally treated with parecoxib (black bars) or vehicle (white bars) by daily i.p. injections throughout an observation period of 7 and 28 days. Data are expressed as means \pm SEM. $n=9$ parecoxib-treated mice (I, J, L, and $\mathbf{M}) ; n=9$ to 10 vehicle-treated mice (I, J, $\mathbf{L}$, and $\mathbf{M})$. ${ }^{*} P<0.05$ versus vehicle. Scale bars: $20 \mu \mathrm{m}$ (A-D); $25 \mu \mathrm{m}$ (E-H); $16 \mu \mathrm{m}$ (K). d, days.
(Figure $1 \mathrm{~F}$ ). ${ }^{26}$ The mice were anesthetized with $2 \%$ isoflurane in oxygen and fixed in supine position on a heated stage, and the abdomen was chemically depilated (Nair hair removal lotion; Church \& Dwight Canada Corp., Mississauga, ON, Canada). A three-dimensional reconstruction and analysis software from VisualSonics (Vevo 770 version 2.3.0) was used to analyze the ultrasound images. To measure the overall volume of developing endometriotic lesions as well as the volume of their stromal tissue and cysts by manual image segmentation, boundaries of the lesions and their cysts were outlined in parallel slices with a step size of $200 \mu \mathrm{m} .^{27}$ Moreover, the growth of lesions and stromal tissue was calculated and the cyst-lesion ratio assessed.

At the end of the in vivo experiments, the anesthetized animals were carefully laparotomized under a stereomicroscope and the largest diameter (D1) and perpendicularly aligned diameter (D2) of the endometriotic lesions were measured by means of a digital caliper. The lesion size (S) was then calculated as follows: $\mathrm{S}=\mathrm{D} 1 \times \mathrm{D} 2 \times \pi / 4{ }^{28}$

\section{Histologic Analysis and Immunohistochemistry}

Formalin-fixed specimens of endometriotic lesions and uterine horns were embedded in paraffin. Sections $(3 \mu \mathrm{m}$ thick) were cut and stained with hematoxylin and eosin according to standard procedures.

For the immunohistochemical detection of $\mathrm{GFP}^{+}$EPCs incorporated into the $\mathrm{GFP}^{-}$microvascular endothelium of the endometriotic lesions, a goat polyclonal anti-GFP antibody (1:50; Rockland Immunochemicals Inc., Limerick, PA) was used as primary antibody. The tissue sections were incubated with a corresponding secondary biotinylated antibody followed by avidin-peroxidase (1:50; Sigma-Aldrich). 3-Amino-9-ethylcarbazole (AEC Substrate System; Abcam, Cambridge, UK) was used as chromogen, and counterstaining was performed with Hemalaun. The fraction of $\mathrm{GFP}^{+}$EPCs in the endometriotic lesions was quantitatively assessed by light microscopy (BX60; Olympus). 
d7
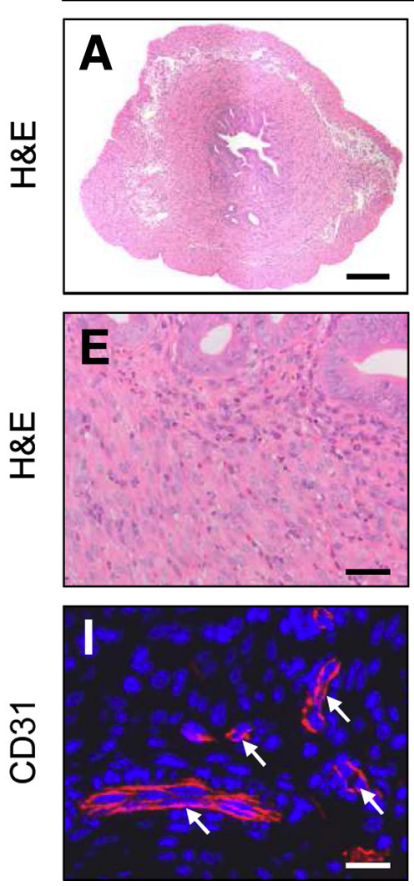
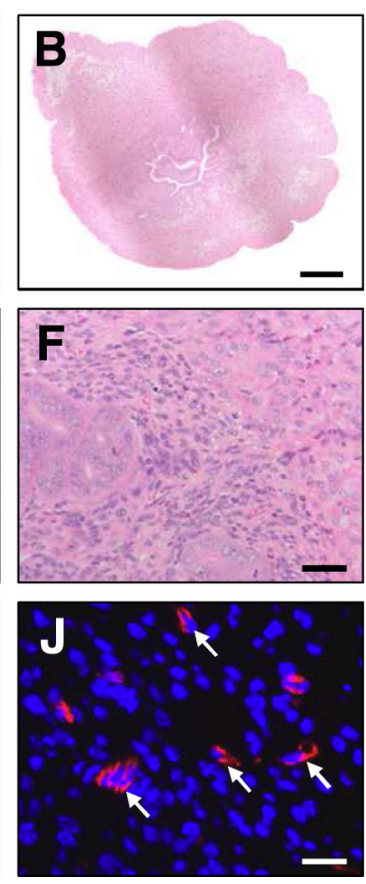

d28
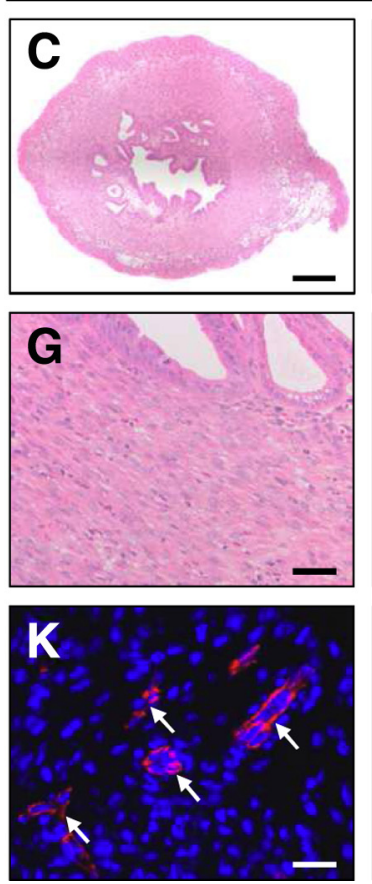
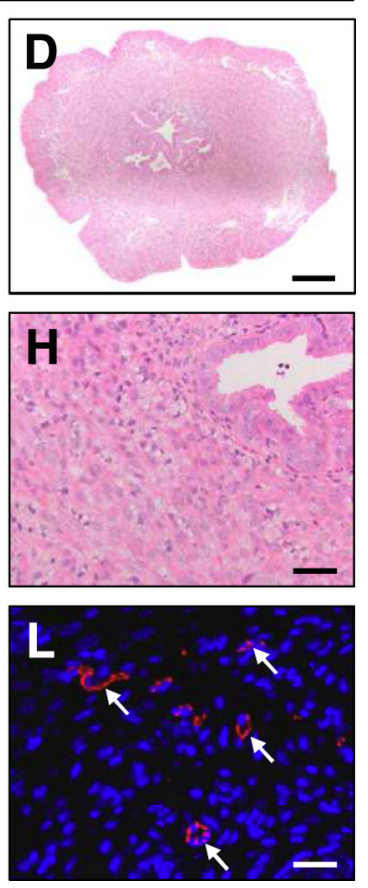

Figure 4 A-H: Hematoxylin and eosin (H\&E)-stained uterine horns of parecoxib- (B, D, F, and $\mathbf{H})$ and vehicle-treated $(\mathbf{A}, \mathbf{C}, \mathbf{E}$, and $\mathbf{G})$ bone marrow-reconstituted FVB/N mice exhibiting comparable morphologic features of the eutopic endometrium at days $7(\mathbf{A}, \mathbf{B}, \mathbf{E}$, and $\mathbf{F})$ and $28(\mathbf{C}, \mathbf{D}, \mathbf{G}$, and $\mathbf{H})$ after induction of endometriotic lesions in the peritoneal cavity. I-L: Immunofluorescent detection of microvessels (arrows) within the uterine horns of parecoxib- (J and $\mathbf{L}$ ) and vehicle-treated ( $\mathbf{I}$ and $\mathbf{K}$ ) bone marrow-reconstituted FVB/N mice exhibiting a comparable vascularization of the eutopic endometrium at days 7 ( $\mathbf{I}$ and $\mathbf{J})$ and $28(\mathbf{K}$ and $\mathbf{L})$ after induction of endometriotic lesions in the peritoneal cavity. Scale bars: $300 \mu \mathrm{m}(\mathbf{A}-\mathbf{D}) ; 40 \mu \mathrm{m}(\mathbf{E}-\mathbf{H}) ; 20 \mu \mathrm{m}(\mathbf{I}-\mathbf{L})$. d, days.

For the immunofluorescent detection of microvessels, sections were stained with a monoclonal rat anti-mouse antibody against the endothelial cell marker CD31 (1:300; Dianova, Hamburg, Germany). A goat anti-rat IgG cyanine 3 antibody (Dianova) served as secondary antibody. Cell nuclei were stained with Hoechst 33342 (1:500; SigmaAldrich). The microvessel density was measured using a BZ-8000 microscope (Keyence, Osaka, Japan). For this purpose, the overall number of CD31-positive microvessels within an endometriotic lesion was counted and divided by the area of stromal lesion tissue.

For the immunohistochemical detection of $\mathrm{COX}-2^{+}$cells as well as proliferating and apoptotic cells in the stroma of the endometriotic lesions, sections were stained with a rabbit polyclonal antibody against COX-2 (1:100; Abcam), a rabbit polyclonal antibody against the proliferation marker Ki-67 (1:100; Abcam), and a rabbit polyclonal antibody against the apoptosis marker cleaved caspase (Casp)-3 (1:100; Cell Signaling, Danvers, MA). Additional sections were stained with antibodies against the lymphocyte marker CD3 (1:100; Abcam), the neutrophilic granulocyte marker myeloperoxidase (MPO) (1:100; Abcam), and the macrophage marker CD68 (1:50; Abcam). A goat anti-rabbit biotinylated antibody (ready to use; Abcam) served as secondary antibody followed by avidin-peroxidase (1:50; Sigma-Aldrich). 3-Amino-9ethylcarbazole (AEC Substrate System; Abcam) was used as chromogen, and counterstaining was performed with
Hemalaun. The fraction of $\mathrm{COX}-2^{+}$cells and proliferating and apoptotic stromal cells and the number of $\mathrm{CD}^{+}$lymphocytes, $\mathrm{MPO}^{+}$neutrophilic granulocytes, and $\mathrm{CD} 68^{+}$macrophages were assessed by counting the numbers of positive cells in four regions of interest within the endometriotic lesions.

\section{Flow Cytometry}

To analyze whether the inhibition of COX-2 also affects the number of circulating EPCs in the bloodstream, flow cytometric analyses of blood samples from parecoxib- and vehicletreated mice were performed. ${ }^{14}$ After lysis of red blood cells and Fc blockade (CD16/CD32; BD Pharmingen, Heidelberg, Germany), the lymphocyte population was analyzed for the expression of the EPC marker stem cell antigen-1fluorescein isothiocyanate (BD Pharmingen) and VEGF receptor (VEGFR)-2-phycoerythrin (BD Pharmingen). Isotype-identical antibodies served as controls (rat IgG2aкfluorescein isothiocyanate-phycoerythrin; BD Pharmingen). Two-color flow cytometric analyses were performed by means of a FACScan (BD Pharmingen). Data were evaluated by the software package CellQuestPro version 3.2 (BD Pharmingen).

\section{Experimental Protocol}

In a first set of experiments, a total of 36 uterine tissue samples were transplanted in $18 \mathrm{GFP}^{+} / \mathrm{Tie}^{+}$bone 
d7
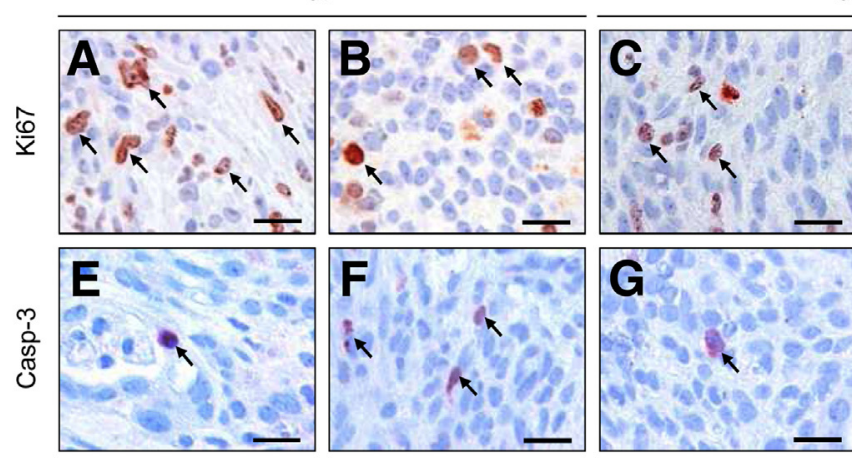

d28
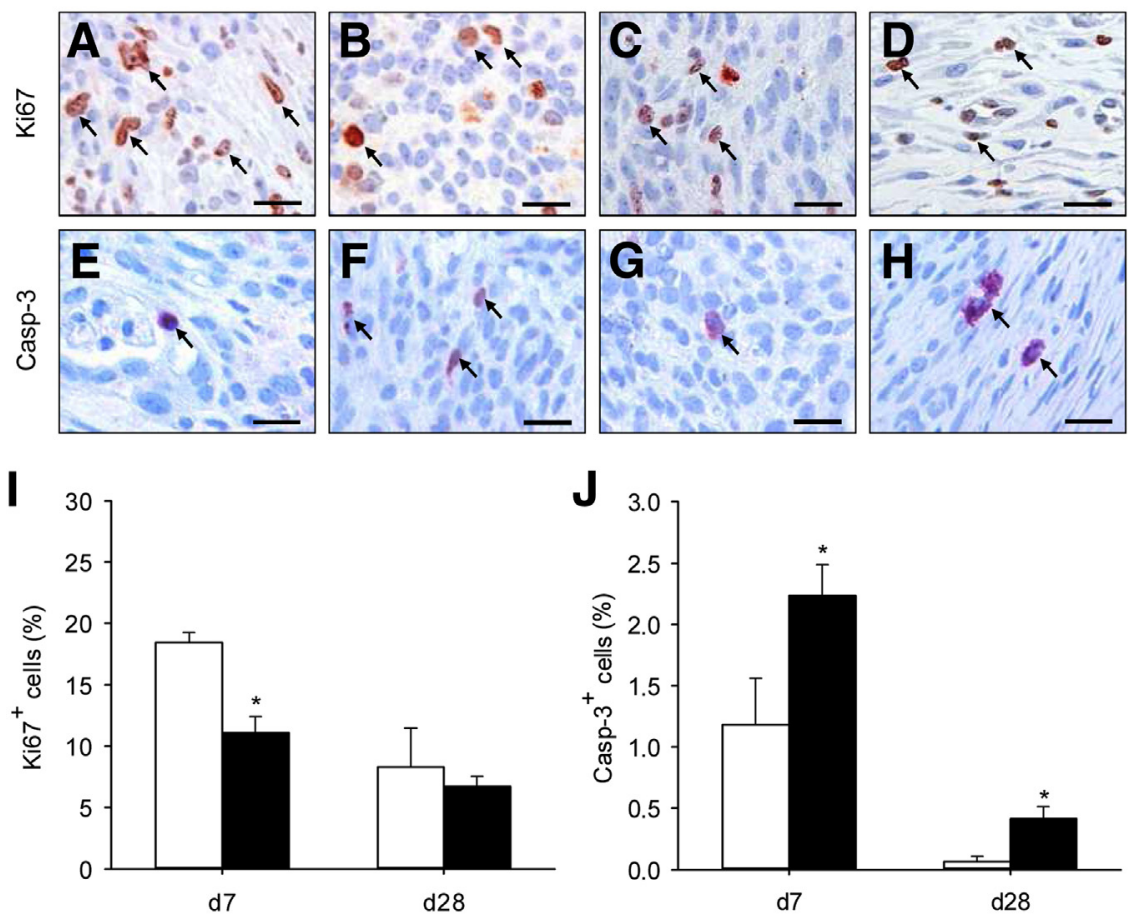

Figure 5 A-H: Immunohistochemical detection of proliferating $\mathrm{Ki}-67^{+}$cells (A-D, arrows) and apoptotic caspase (Casp) $-3^{+}$cells ( $\mathbf{E}-\mathbf{H}$, arrows) in the stroma of endometriotic lesions at day 7 (A, B, E, and $\mathbf{F})$ and 28 (C, D, G, and $\mathbf{H})$ after transplantation of uterine tissue samples into the peritoneal cavity of bone marrow-reconstituted FVB/N mice, which received $\beta$-estradiol 17valerate once a week and were additionally treated with parecoxib (B, D, F, and $\mathbf{H})$ or vehicle (A, C, E, and $\mathbf{G}$ ) by daily i.p. injections. I and J: Ki$67^{+}$cells $(\mathrm{I})$ and Casp- $3^{+}$cells $(\mathrm{J})$ in the stroma of endometriotic lesions of bone marrowreconstituted FVB/N mice, which received $\beta$-estradiol 17-valerate once a week and were additionally treated with parecoxib (black bars) or vehicle (white bars) by daily i.p. injections throughout an observation period of 7 and 28 days. Data are expressed as means \pm SEM. $n=9$ parecoxib-treated mice (I and J); $n=9$ to 10 vehicle-treated mice (I and $\mathbf{J}) .{ }^{*} P<0.05$ versus vehicle. Scale bars $=20 \mu \mathrm{m}(\mathbf{A}-\mathbf{H})$. d, days. marrow-reconstituted animals, which received a single s.c. injection of $100 \mu \mathrm{g} / \mathrm{kg}$ of $\beta$-estradiol 17-valerate. The mice were randomly divided into two groups $(n=9$ each), which were treated with $25 \mathrm{mg} / \mathrm{kg}$ parecoxib or vehicle (sodium chloride; control) by daily i.p. injections. After 7 days, tissue and blood samples were harvested and further processed for histologic analysis, immunohistochemical analysis, and flow cytometry.

In a second set of experiments, a total of 38 uterine tissue samples were transplanted in $19 \mathrm{GFP}^{+} / \mathrm{Tie}^{+}$bone marrow-reconstituted animals. The mice were randomly divided into two groups receiving $100 \mu \mathrm{g} / \mathrm{kg}$ s.c. $\beta$-estradiol 17 -valerate once a week and $25 \mathrm{mg} / \mathrm{kg}$ of parecoxib $(n=9)$ or vehicle (control; $n=10$ ) by daily i.p. injections for 28 days. Ultrasound image analyses of the endometriotic lesions were performed directly after tissue transplantation (day 0) as well as at days $7,14,21$, and 28 . At the end of the experiments, the size of the endometriotic lesions was additionally assessed by means of a digital caliper. Tissue and blood samples were taken and further processed for histologic analysis, immunohistochemical analysis, and flow cytometry.

\section{Statistical Analysis}

Data were first analyzed for normal distribution and equal variance. In case of parametric data, differences between the two experimental groups were assessed by the unpaired $t$-test. In case of nonparametric data, differences between the two experimental groups were assessed by the MannWhitney rank sum test. To test for time effects within each experimental group, analysis of variance for repeated measurements was applied followed by the Dunnett post hoc test (SigmaPlot 13.0; Jandel Corporation, San Rafael, CA). All data are expressed as means \pm SEM. Statistical significance was accepted for $P<0.05$.

\section{Results}

\section{Growth and Cyst Formation of Endometriotic Lesions}

The growth and cyst formation of developing endometriotic lesions were repetitively analyzed by means of high-resolution ultrasonography for 28 days (Figure 2, A and B). Lesions of vehicle- and parecoxib-treated animals exhibited a comparable initial size of $1.2 \mathrm{~mm}^{3}$ at day 0 (Figure 2C). Throughout the following observation period, the growth of parecoxib-treated endometriotic lesions was markedly suppressed, resulting in a significantly lower overall lesion volume of approximately 2 $\mathrm{mm}^{3}$ at day 28 when compared with vehicle-treated controls (approximately $6 \mathrm{~mm}^{3}$ ) (Figure 2, A-D). More detailed analyses of the stromal and cyst-like dilated glandular parts of the lesions revealed that this was caused by an inhibition of stromal tissue proliferation during the early development of the lesions within the first 14 days after surgical induction (Figure 2, E and F). Moreover, parecoxib-treated lesions exhibited a reduced secretory activity between days 14 and 28, as indicated by significantly lower cyst volumes and cyst/lesion ratios (Figure 2, G and $\mathrm{H}$ ).

These results were confirmed by additional histologic analyses and caliper measurements of the lesions at the end of the 
in vivo experiments. As expected, parecoxib-treated lesions contained smaller cyst-like dilated glands when compared with vehicle-treated controls (Figure 2, I and J). In addition, their overall size was significantly reduced (Figure $2 \mathrm{~K}$ ).

\section{COX-2 Expression and Vascularization of Endometriotic} Lesions

Immunohistochemical analyses revealed that COX-2 expression was particularly high within the stroma of vehicle-treated control lesions at day 7 (Figure 3, A-D and I). Parecoxib treatment significantly reduced this enhanced expression of COX-2 in the initial phase of engraftment (Figure 3, B and I).

The engraftment of spread endometrial tissue at ectopic sites is associated with the formation of new microvascular networks via angiogenesis and vasculogenesis. ${ }^{2,12}$ Additional immunohistochemical analyses of the newly developing endometriotic lesions found that these two processes were also suppressed by the inhibition of COX-2. Parecoxibtreated lesions exhibited a significantly reduced microvessel density at day 7 but not at day 28 when compared with vehicle-treated controls (Figure 3, E-H and J). Previous findings indicate that there is a positive correlation between lesion vascularization and numbers of recruited EPCs. ${ }^{11}$ Accordingly, the microvascular endothelium of parecoxibtreated lesions also contained less incorporated $\mathrm{GFP}^{+}$EPCs at day 7 (Figure 3, K and L). Of interest, this was still the case at day 28 (Figure $3 \mathrm{~L}$ ). The numbers of circulating stem cell antigen- $1^{+}$/VEGFR-2 ${ }^{+}$EPCs were increased in the bloodstream of parecoxib-treated animals when compared with vehicle-treated controls by flow cytometry (Figure 3M).

Besides, histologic and immunohistochemical analyses of the eutopic endometrium within the uterine horns of parecoxib- and vehicle-treated animals were performed. These analyses revealed that treatment with the COX-2 inhibitor affected neither the morphologic features nor the vascularization of the tissue (Figure 4).

\section{Cell Proliferation and Apoptotic Cell Death in Endometriotic Lesions}

A sufficient vascularization is the major prerequisite for the proliferation and survival of endometriotic lesions. Accordingly, a significantly lower number of proliferating $\mathrm{Ki}-67^{+}$cells were detected in the endometrial stroma of parecoxib-treated lesions at day 7 but not at day 28 when compared with vehicle-treated controls (Figure 5, A-D and I). In addition, parecoxib-treated lesions contained more apoptotic Casp- $3^{+}$cells at days 7 and 28 (Figure 5, E-H and $\mathrm{J}$ ).

\section{Immune Cell Infiltration into Endometriotic Lesions}

Because the COX-2 signaling pathway is a major regulator of inflammatory processes, ${ }^{29}$ immune cell infiltration into the endometriotic lesions was analyzed. Immunohistochemical staining of different immune cell subpopulations revealed that the lesions were primarily infiltrated by $\mathrm{CD}^{+} 8^{+}$macrophages and $\mathrm{MPO}^{+}$neutrophilic granulocytes, whereas only a few infiltrating $\mathrm{CD}^{+}$lymphocytes could be detected within the endometrial stroma (Figure 6, A-L). Treatment with parecoxib suppressed this immune cell infiltration, as indicated by significantly reduced numbers of $\mathrm{CD}^{+} 8^{+}$macrophages and $\mathrm{MPO}^{+}$neutrophilic granulocytes when compared with vehicle-treated controls (Figure 6, M-O).

\section{Discussion}

An increasing number of studies suggest the inhibition of COX-2 signaling for the treatment of angiogenesis-related diseases. ${ }^{30-32}$ This therapeutic approach has also been proven to be successful in preclinical endometriosis models. Machado et $\mathrm{al}^{33}$ reported that parecoxib treatment downregulates the expression of VEGF and its receptor Flk-1, which results in a reduced vascularization and growth of endometriotic lesions in rats. Potent anti-VEGF effects on endometriosis have also been observed with the COX-2 inhibitor NS398 in vitro and in vivo. ${ }^{23,24,34}$ Moreover, Jana et $\mathrm{al}^{15}$ recently reported that suppression of COX-2/PGE $2 /$ phospho-AKT signaling attenuates matrix metalloproteinase-2-mediated angiogenesis in murine endometriotic lesions. In the present study, we provide evidence that vasculogenesis is another important COX-2-regulated process that contributes to blood vessel formation in endometriosis. In fact, treatment with parecoxib inhibits the incorporation of circulating EPCs in the microvasculature of endometriotic lesions and, thus, affects their vascularization in the initial phase of engraftment.

For the in vivo experiments, a previously established murine $\mathrm{GFP}^{+} / \mathrm{GFP}^{-}$crossover design model was used. ${ }^{14}$ This model involves the transplantation of $\mathrm{GFP}^{+}$bone marrow from transgenic FVB/N-TgN (Tie2/GFP) 287 Sato mice into irradiated FVB/N wild-type animals. Importantly, we recently found that this procedure leads to low estrogen levels in the recipients, which are similar to those measured in ovariectomized mice. ${ }^{14}$ Accordingly, we substituted the animals with $\beta$-estradiol 17 -valerate once a week to analyze the effect of COX-2 inhibition on the homing of EPCs in endometriotic lesions under physiologic estrogen levels. For this purpose, the lesions were surgically induced in the peritoneal cavity by fixation of uterine tissue samples to the abdominal wall. This approach allowed the repetitive analysis of developing endometriotic lesions by means of $40-\mathrm{MHz}$ high-resolution ultrasonography. In contrast to conventional approaches for the assessment of lesion sizes, such as caliper and weight measurements, ${ }^{35-37}$ this technology bears the major advantage that stromal and cyst-like dilated glandular parts of the lesions can be easily identified and separately analyzed. ${ }^{26}$ Hence, it is possible to clearly differentiate between stromal tissue growth and enhanced 
d7
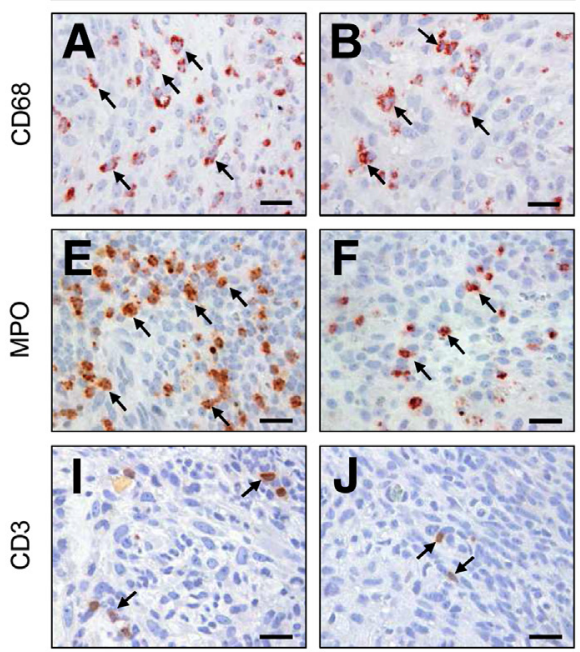

M
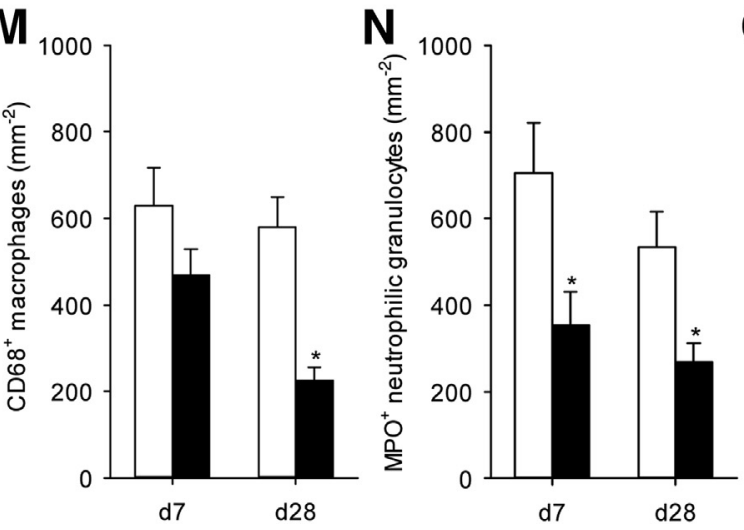

d28
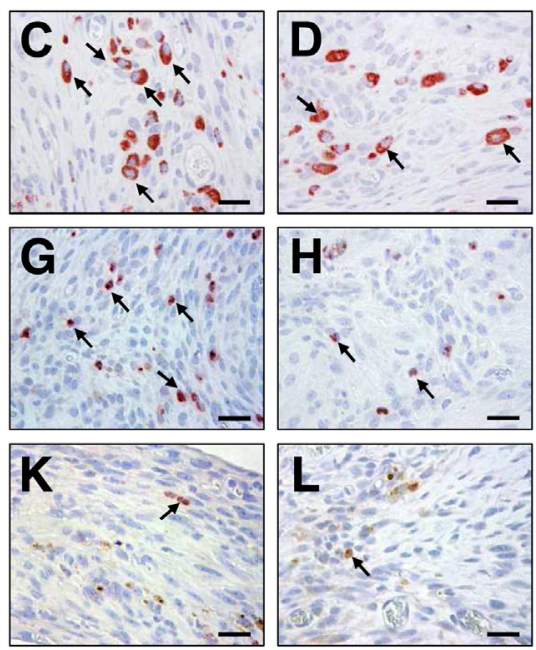

0

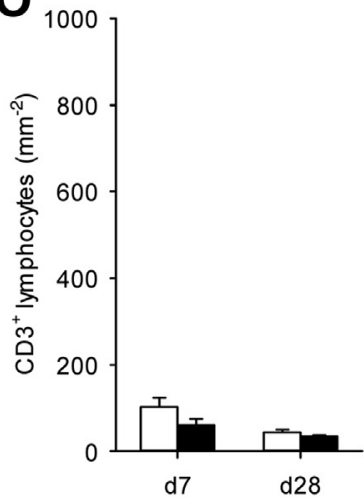

Figure 6 A-L: Immunohistochemical detection of $\mathrm{CD} 68^{+}$macrophages (A-D, arrows), myeloperoxidase $(\mathrm{MPO})^{+}$neutrophilic granulocytes (E-H, arrows), and $\mathrm{CD}^{+}$lymphocytes (I-L, arrows) in the stroma of endometriotic lesions at days 7 (A, B, E, F, I, and J) and 28 (C, D, G, H, K, and $\mathbf{L})$ after transplantation of uterine tissue samples into the peritoneal cavity of bone marrow-reconstituted FVB/N mice, which received $\beta$-estradiol 17-valerate once a week and were additionally treated with parecoxib $(\mathbf{B}, \mathbf{D}, \mathbf{F}, \mathbf{H}, \mathbf{J}$, and $\mathbf{L}$ ) or vehicle $(\mathbf{A}, \mathbf{C}, \mathbf{E}, \mathbf{G}, \mathbf{I}$, and $\mathbf{K})$ by daily i.p. injections. $\mathrm{M}-\mathbf{0}$ : $\mathrm{CD}^{+} 8^{+}$macrophages (M), $\mathrm{MPO}^{+}$ neutrophilic granulocytes $(\mathbf{N})$, and $\mathrm{CD}^{+}$lymphocytes (0) in the stroma of endometriotic lesions of bone marrow-reconstituted FVB/N mice, which received $\beta$-estradiol 17 -valerate once a week and were additionally treated with parecoxib (black bars) or vehicle (white bars) by daily i.p. injections throughout an observation period of 7 and 28 days. Data are expressed as means \pm SEM. $n=9$ parecoxib-treated mice $(\mathbf{M}-\mathbf{0}) ; n=9$ to 10 vehicle-treated mice $(\mathbf{M}-\mathbf{0})$. ${ }^{*} P<0.05$ versus vehicle. Scale bars $=20 \mu \mathrm{m}(\mathbf{A}-\mathbf{L})$. $d$, days. glandular secretory activity, which both can contribute to an increase of lesion size over time.

The ultrasound analyses revealed that stromal tissue growth was suppressed in parecoxib-treated lesions during the first 14 days after surgical induction. Additional immunohistochemical analyses found that this was associated with a significantly reduced expression of COX-2 at day 7 when compared with vehicle-treated controls. This observation is in line with previous studies reporting that parecoxib not only inhibits the activity of COX-2 but also suppresses the expression of the enzyme. ${ }^{38,39}$

In the initial phase of engraftment, ectopic endometrial tissue is particularly dependent on the rapid establishment of a functional microvasculature to guarantee a sufficient oxygen and nutrient supply, ${ }^{40,41}$ which is a major prerequisite for cell survival and proliferative activity. However, the early vascularization of the lesions was impaired in parecoxib-treated animals, as indicated by a significantly lower microvessel density at day 7 when compared with vehicle-treated controls. This finding may explain the lower number of $\mathrm{Ki}-67^{+}$proliferating cells and higher number of Casp $-3^{+}$apoptotic cells within the lesions at this early time point. On the other hand, inhibition of COX-2 directly affects proliferation and viability of malignant and benign cells and tissues. ${ }^{42-44}$ Accordingly, parecoxib-treated lesions still exhibited increased numbers of apoptotic cells at day 28. It may be assumed that the engraftment of the lesions, which is associated with a high vascularization activity and cell turnover, was finally completed in the two experimental groups at this late time point. Hence, although a delayed vascularization was detected under COX-2 treatment, the lesions of the two groups no longer differed in terms of microvessel density and numbers of proliferating $\mathrm{Ki}-67^{+}$cells. Nonetheless, even at day 28, a significantly reduced number of EPCs were detected in the microvasculature of parecoxib-treated lesions when compared with vehicle-treated controls. This finding suggests that the continuous self-renewal process of the microvascular endothelium via the incorporation of circulating EPCs was still impaired by parecoxib treatment in this late phase of lesion development, which is particularly characterized by microvascular network maturation and remodeling. ${ }^{14}$

Of interest, blockade of $\mathrm{COX}-2 / \mathrm{PGE}_{2}$ signaling was found to reduce the secretory activity of endometrial glands, as indicated by a markedly decreased cyst volume and cyst/ lesion ratio of parecoxib-treated lesions between days 21 
and 28. In line with this result, COX-2 seems to be crucially involved in the regulation of water-transporting aquaporins. ${ }^{45}$ In addition, $\mathrm{PGE}_{2}$ has been reported to stimulate other secretory processes. ${ }^{46-48}$

The observed inhibitory action of parecoxib on vasculogenesis in endometriotic lesions may be attributable to different effects on EPCs. Colleselli et $\mathrm{al}^{18}$ reported that inhibition of COX-2 causes a significant reduction of EPC proliferation by inducing apoptosis and cell cycle arrest. This direct effect on the viability of EPCs may also affect their incorporation into newly developing microvessels. In addition, COX-2 inhibition may interfere with trafficking mechanisms that mediate the mobilization of EPCs from the bone marrow into the bloodstream and their subsequent recruitment in endometriotic lesions. Decreased stromal cell-derived factor (SDF)-1 levels in the bone marrow increase the mobilization of EPCs and other stem cells into the bloodstream. ${ }^{49,50}$ On the other hand, suppression of the SDF-1/chemokine receptor type 4 axis also inhibits the recruitment of EPCs in endometriotic lesions. ${ }^{11,13}$ Considering the fact that COX-2 inhibition down-regulates SDF-1 expression ${ }^{51}$ these findings fit well with our observation that the number of circulating EPCs was significantly higher, whereas the number of EPCs incorporated into the microvascular endothelium of endometriotic lesions was significantly lower in parecoxib-treated mice when compared with vehicle-treated controls.

Finally, it is well known that endometriotic lesions generate a proinflammatory environment in the peritoneal cavity, which may also be a major cause for endometriosisassociated pain. ${ }^{52}$ Accordingly, targeting COX-2 is a promising approach to suppress different inflammatory mechanisms, such as the production of proinflammatory cytokines and the activation of immune cells. ${ }^{53,54}$ Therefore, we additionally analyzed in this study the effect of parecoxib on immune cell infiltration in endometriotic lesions. Of interest, the endometriotic lesions were mainly infiltrated by macrophages and neutrophilic granulocytes, whereas only a few lymphocytes could be detected in the endometrial stromal tissue. The latter observation contradicts the results of previous studies that reported that lymphocytes are a major immune cell subpopulation in murine and human endometriotic lesions. ${ }^{55,56}$ However, in the present experimental setting, the analyzed mice underwent irradiation and bone marrow transplantation. Hence, these interventions may change the ratios of individual immune cell subsets or their response to the ectopic endometrial tissue in the peritoneal cavity.

Taken together, the present study found that inhibition of COX-2 suppresses the recruitment of EPCs in the microvasculature of endometriotic lesions. Therefore, COX-2 inhibitors may be suitable to suppress vasculogenesis in endometriosis. Besides the well-known antiangiogenic, antiproliferative, anti-inflammatory, analgesic, and proapoptotic activity of COX-2 inhibitors, this novel mechanism may further contribute to their beneficial action profile in the treatment of endometriosis. Such a treatment may be particularly effective to prevent the formation of early engrafting red lesions, which are characterized by a high vascularization rate and many immature microvessels. ${ }^{4,57}$ In contrast, older black and white lesions that exhibit fewer microvessels and more fibrous tissue may be more resistant. Moreover, therapeutic approaches that target the formation of new blood vessels in endometriotic lesions may also affect physiologic angiogenesis in the reproductive organs. Our observation that the vascularization of the eutopic endometrium in the uterus was comparable in parecoxiband vehicle-treated animals indicates that this was not the case in the present study. However, additional studies analyzing in more detail the effects of COX-2 inhibitors on the female reproductive system are needed to clarify in which phases of endometriosis therapy these drugs may be useful without inducing severe adverse effects.

\section{Acknowledgments}

We thank Janine Becker, Sandra Schuler, and Caroline Bickelmann (Institute for Clinical \& Experimental Surgery, Homburg/Saar, Germany) for technical assistance.

\section{References}

1. Giudice LC: Clinical practice: endometriosis. N Engl J Med 2010, 362:2389-2398

2. Groothuis PG, Nap AW, Winterhager E, Grümmer R: Vascular development in endometriosis. Angiogenesis 2005, 8:147-156

3. Laschke MW, Menger MD: In vitro and in vivo approaches to study angiogenesis in the pathophysiology and therapy of endometriosis. Hum Reprod Update 2007, 13:331-342

4. Nisolle M, Casanas-Roux F, Anaf V, Mine JM, Donnez J: Morphometric study of the stromal vascularization in peritoneal endometriosis. Fertil Steril 1993, 59:681-684

5. Rocha AL, Reis FM, Taylor RN: Angiogenesis and endometriosis Obstet Gynecol Int 2013, 2013:859619

6. Laschke MW, Menger MD: Anti-angiogenic treatment strategies for the therapy of endometriosis. Hum Reprod Update 2012, 18:682-702

7. Djokovic D, Calhaz-Jorge C: Angiogenesis as a therapeutic target in endometriosis. Acta Med Port 2014, 27:489-497

8. Laschke MW, Elitzsch A, Vollmar B, Menger MD: In vivo analysis of angiogenesis in endometriosis-like lesions by intravital fluorescence microscopy. Fertil Steril 2005, 84 Suppl 2:1199-1209

9. Filippi I, Carrarelli P, Luisi S, Batteux F, Chapron C, Naldini A, Petraglia F: Different expression of hypoxic and angiogenic factors in human endometriotic lesions. Reprod Sci 2016, 23:492-497

10. Becker CM, Beaudry P, Funakoshi T, Benny O, Zaslavsky A, Zurakowski D, Folkman J, D'Amato RJ, Ryeom S: Circulating endothelial progenitor cells are up-regulated in a mouse model of endometriosis. Am J Pathol 2011, 178:1782-1791

11. Laschke MW, Giebels C, Nickels RM, Scheuer C, Menger MD Endothelial progenitor cells contribute to the vascularization of endometriotic lesions. Am J Pathol 2011, 178:442-450

12. Laschke MW, Giebels C, Menger MD: Vasculogenesis: a new piece of the endometriosis puzzle. Hum Reprod Update 2011, 17:628-636

13. Virani S, Edwards AK, Thomas R, Childs T, Tayade C: Blocking of stromal cell-derived factor-1 reduces neoangiogenesis in human endometriosis lesions in a mouse model. Am J Reprod Immunol 2013, 70:386-397 
14. Rudzitis-Auth J, Nenicu A, Nickels RM, Menger MD, Laschke MW: Estrogen stimulates homing of endothelial progenitor cells to endometriotic lesions. Am J Pathol 2016, 186:2129-2142

15. Jana S, Chatterjee K, Ray AK, DasMahapatra P, Swarnakar S: Regulation of matrix metalloproteinase-2 activity by COX-2-PGE2pAKT axis promotes angiogenesis in endometriosis. PLoS One 2016, 11:e0163540

16. Olivares CN, Alaniz LD, Menger MD, Barañao RI, Laschke MW, Meresman GF: Inhibition of hyaluronic acid synthesis suppresses angiogenesis in developing endometriotic lesions. PLoS One 2016, 11:e0152302

17. Yang RQ, Teng H, Xu XH, Liu SY, Wang YH, Guo FJ, Liu XJ: Microarray analysis of microRNA deregulation and angiogenesisrelated proteins in endometriosis. Genet Mol Res 2016, 15. gmr. 15027826

18. Colleselli D, Bijuklic K, Mosheimer BA, Kähler CM: Inhibition of cyclooxygenase (COX)-2 affects endothelial progenitor cell proliferation. Exp Cell Res 2006, 312:2933-2941

19. Ota H, Igarashi S, Sasaki M, Tanaka T: Distribution of cyclooxygenase- 2 in eutopic and ectopic endometrium in endometriosis and adenomyosis. Hum Reprod 2001, 16:561-566

20. Chishima F, Hayakawa S, Sugita K, Kinukawa N, Aleemuzzaman S, Nemoto $\mathrm{N}$, Yamamoto $\mathrm{T}$, Honda $\mathrm{M}$ : Increased expression of cyclooxygenase-2 in local lesions of endometriosis patients. Am J Reprod Immunol 2002, 48:50-56

21. Cobellis L, Razzi S, De Simone S, Sartini A, Fava A, Danero S, Gioffrè W, Mazzini M, Petraglia F: The treatment with a COX-2 specific inhibitor is effective in the management of pain related to endometriosis. Eur J Obstet Gynecol Reprod Biol 2004, 116:100-102

22. Nasir L, Bope ET: Management of pelvic pain from dysmenorrhea or endometriosis. J Am Board Fam Pract 2004, 17 Suppl:S43-S47

23. Ozawa Y, Murakami T, Tamura M, Terada Y, Yaegashi N, Okamura K: A selective cyclooxygenase-2 inhibitor suppresses the growth of endometriosis xenografts via antiangiogenic activity in severe combined immunodeficiency mice. Fertil Steril 2006, 86: $1146-1151$

24. Laschke MW, Elitzsch A, Scheuer C, Vollmar B, Menger MD: Selective cyclo-oxygenase-2 inhibition induces regression of autologous endometrial grafts by down-regulation of vascular endothelial growth factor-mediated angiogenesis and stimulation of caspase-3-dependent apoptosis. Fertil Steril 2007, 87:163-171

25. Committee for the Update of the Guide for the Care and Use of Laboratory Animals; National Research Council: Guide for the Care and Use of Laboratory Animals: Eighth Edition. Washington, DC, National Academies Press, 2011

26. Laschke MW, Körbel C, Rudzitis-Auth J, Gashaw I, Reinhardt M, Hauff P, Zollner TM, Menger MD: High-resolution ultrasound imaging: a novel technique for the noninvasive in vivo analysis of endometriotic lesion and cyst formation in small animal models. Am J Pathol 2010, 176:585-593

27. Rudzitis-Auth J, Menger MD, Laschke MW: Resveratrol is a potent inhibitor of vascularization and cell proliferation in experimental endometriosis. Hum Reprod 2013, 28:1339-1347

28. Becker CM, Rohwer N, Funakoshi T, Cramer T, Bernhardt W, Birsner A, Folkman J, D'Amato RJ: 2-methoxyestradiol inhibits hypoxia-inducible factor-1 1 alpha\} and suppresses growth of lesions in a mouse model of endometriosis. Am J Pathol 2008, 172:534-544

29. Yu T, Lao X, Zheng H: Influencing COX-2 activity by COX related pathways in inflammation and cancer. Mini Rev Med Chem 2016, 16: $1230-1243$

30. Sennlaub F, Valamanesh F, Vazquez-Tello A, El-Asrar AM, Checchin D, Brault S, Gobeil F, Beauchamp MH, Mwaikambo B, Courtois Y, Geboes K, Varma DR, Lachapelle P, Ong H, BeharCohen F, Chemtob S: Cyclooxygenase-2 in human and experimental ischemic proliferative retinopathy. Circulation 2003, 108:198-204

31. Youn J, Cho ML, Kim YJ, Yun HS, Park SH, Jin CZ, Paik DJ, Kim HY: New cyclooxygenase-2 inhibitor DFU regulates vascular endothelial growth factor expression in rheumatoid synoviocytes. Immunol Lett 2005, 96:219-224

32. Sui W, Zhang Y, Wang Z, Wang Z, Jia Q, Wu L, Zhang W: Antitumor effect of a selective COX-2 inhibitor, celecoxib, may be attributed to angiogenesis inhibition through modulating the PTEN/PI3K/Akt/HIF-1 pathway in an $\mathrm{H}_{22}$ murine hepatocarcinoma model. Oncol Rep 2014, 31:2252-2260

33. Machado DE, Berardo PT, Landgraf RG, Fernandes PD, Palmero C, Alves LM, Abrao MS, Nasciutti LE: A selective cyclooxygenase-2 inhibitor suppresses the growth of endometriosis with an antiangiogenic effect in a rat model. Fertil Steril 2010, 93:2674-2679

34. Huang F, Cao J, Liu Q, Zou Y, Li H, Yin T: MAPK/ERK signal pathway involved expression of COX-2 and VEGF by IL-1 $\beta$ induced in human endometriosis stromal cells in vitro. Int J Clin Exp Pathol 2013, 6:2129-2136

35. Rudzitis-Auth J, Körbel C, Scheuer C, Menger MD, Laschke MW: Xanthohumol inhibits growth and vascularization of developing endometriotic lesions. Hum Reprod 2012, 27:1735-1744

36. Wang CC, Xu H, Man GC, Zhang T, Chu KO, Chu CY, Cheng JT, Li G, He YX, Qin L, Lau TS, Kwong J, Chan TH: Prodrug of green tea epigallocatechin-3-gallate (Pro-EGCG) as a potent antiangiogenesis agent for endometriosis in mice. Angiogenesis 2013, 16:59-69

37. Long Q, Liu X, Guo SW: Surgery accelerates the development of endometriosis in mice. Am J Obstet Gynecol 2016, 215:320.e1-320. e15

38. Nenicu A, Gu Y, Körbel C, Menger MD, Laschke MW: Combination therapy with telmisartan and parecoxib induces regression of endometriotic lesions. Br J Pharmacol 2017, 174:2623-2635

39. Vértiz-Hernández ÁA, Martínez-Morales F, Valle-Aguilera R, LópezSánchez P, Villalobos-Molina R, Pérez-Urizar J: Parecoxib increases blood pressure through inhibition of cyclooxygenase-2 messenger RNA in an experimental model. Rev Invest Clin 2015, 67:250-257

40. May K, Becker CM: Endometriosis and angiogenesis. Minerva Ginecol 2008, 60:245-254

41. Hey-Cunningham AJ, Peters KM, Zevallos HB, Berbic M, Markham R, Fraser IS: Angiogenesis, lymphangiogenesis and neurogenesis in endometriosis. Front Biosci (Elite Ed.) 2013, 5: 1033-1056

42. Churchman A, Baydoun AR, Hoffman R: Inhibition of angiogenic tubule formation and induction of apoptosis in human endothelial cells by the selective cyclooxygenase-2 inhibitor 5-bromo-2-(4-fluorophenyl)-3-(methylsulfonyl) thiophene (DuP-697). Eur J Pharmacol 2007, 573:176-183

43. Olivares C, Ricci A, Bilotas M, Barañao RI, Meresman G: The inhibitory effect of celecoxib and rosiglitazone on experimental endometriosis. Fertil Steril 2011, 96:428-433

44. Sato A, Mizobuchi Y, Nakajima K, Shono K, Fujihara T, Kageji T, Kitazato K, Matsuzaki K, Mure H, Kuwayama K, Sumi A, Saya H, Sampetrean O, Nagahirao S: Blocking COX-2 induces apoptosis and inhibits cell proliferation via the Akt/survivin- and Akt/ID3 pathway in low-grade-glioma. J Neurooncol 2017, 132:231-238

45. Wang L, Shan Y, Ye Y, Jin L, Zhuo Q, Xiong X, Zhao X, Lin L, Miao J: COX-2 inhibition attenuates lung injury induced by skeletal muscle ischemia reperfusion in rats. Int Immunopharmacol 2016, 31 : $116-122$

46. Muro S, Tanaka I, Usui T, Kotani M, Koide S, Mukoyama M, Fukata J, Itoh H, Narumiya S, Kawata M, Nakao K: Expression of prostaglandin E receptor EP4 subtype in rat adrenal zona glomerulosa: involvement in aldosterone release. Endocr $\mathrm{J}$ 2000, 47: 429-436

47. Sauer JR, Essenberg RC, Bowman AS: Salivary glands in ixodid ticks: control and mechanism of secretion. J Insect Physiol 2000, 46: 1069-1078

48. Mohn CE, Fernandez-Solari J, De Laurentiis A, Prestifilippo JP, de la Cal C, Funk R, Bornstein SR, McCann SM, Rettori V: The rapid release of corticosterone from the adrenal induced by ACTH is 
mediated by nitric oxide acting by prostaglandin E2. Proc Natl Acad Sci U S A 2005, 102:6213-6218

49. Petit I, Szyper-Kravitz M, Nagler A, Lahav M, Peled A, Habler L, Ponomaryov T, Taichman RS, Arenzana-Seisdedos F, Fujii N, Sandbank J, Zipori D, Lapidot T: G-CSF induces stem cell mobilization by decreasing bone marrow SDF-1 and up-regulating CXCR4 . Nat Immunol 2002, 3:687-694

50. De Falco E, Porcelli D, Torella AR, Straino S, Iachininoto MG, Orlandi A, Truffa S, Biglioli P, Napolitano M, Capogrossi MC, Pesce M: SDF-1 involvement in endothelial phenotype and ischemia-induced recruitment of bone marrow progenitor cells. Blood 2004, 104:3472-3482

51. Kim YS, Bigliani LU, Fujisawa M, Murakami K, Chang SS, Lee HJ, Lee FY, Blaine TA: Stromal cell-derived factor 1 (SDF-1, CXCL12) is increased in subacromial bursitis and downregulated by steroid and nonsteroidal anti-inflammatory agents. J Orthop Res 2006, 24:1756-1764

52. McKinnon BD, Bertschi D, Bersinger NA, Mueller MD: Inflammation and nerve fiber interaction in endometriotic pain. Trends Endocrinol Metab 2015, 26:1-10
53. Iezzi A, Ferri C, Mezzetti A, Cipollone F: COX-2: friend or foe? Curr Pharm Des 2007, 13:1715-1721

54. Fan LW, Kaizaki A, Tien LT, Pang Y, Tanaka S, Numazawa S, Bhatt AJ, Cai Z: Celecoxib attenuates systemic lipopolysaccharideinduced brain inflammation and white matter injury in the neonatal rats. Neuroscience 2013, 240:27-38

55. Oosterlynck DJ, Cornillie FJ, Waer M, Koninckx PR: Immunohistochemical characterization of leucocyte subpopulations in endometriotic lesions. Arch Gynecol Obstet 1993, 253:197-206

56. Nenicu A, Körbel C, Gu Y, Menger MD, Laschke MW: Combined blockade of angiotensin II type 1 receptor and activation of peroxisome proliferator-activated receptor- $\gamma$ by telmisartan effectively inhibits vascularization and growth of murine endometriosis-like lesions. Hum Reprod 2014, 29:1011-1024

57. Matsuzaki S, Canis M, Murakami T, Dechelotte P, Bruhat MA, Okamura K: Immunohistochemical analysis of the role of angiogenic status in the vasculature of peritoneal endometriosis. Fertil Steril 2001, 76:712-716 